\title{
Emerging antiviral resistant strains of influenza $A$ and the potential therapeutic targets within the viral ribonucleoprotein (vRNP) complex
}

\author{
Alicia M Davis, Bryan J Chabolla and Laura L Newcomb*
}

\begin{abstract}
Emerging antiviral resistant strains of influenza A virus are greatly limiting the therapies available to stop aggressive infections. Genome changes that confer resistance to the two classes of approved antivirals have been identified in circulating influenza A viruses. It is only a matter of time before the currently approved influenza A antivirals are rendered ineffective, emphasizing the need for additional influenza antiviral therapies. This review highlights the current state of antiviral resistance in circulating and highly pathogenic influenza A viruses and explores potential antiviral targets within the proteins of the influenza A virus ribonucleoprotein (vRNP) complex, drawing attention to the viral protein activities and interactions that play an indispensable role in the influenza life cycle. Investigation of small molecule inhibition, accelerated by the use of crystal structures of VRNP proteins, has provided important information about viral protein domains and interactions, and has revealed many promising antiviral drug candidates discussed in this review.
\end{abstract}

Keywords: Influenza, Resistance, Antiviral, RdRP, NP

\section{Background}

Influenza A viruses are infectious agents spread through contact or aerosol droplets that result in a seasonal respiratory illness which can potentially lead to death. Highly transmissible influenza A viruses can reach pandemic proportions as seen in 1918, 1957, 1968, and 2009. The natural reservoir for influenza A viruses are aquatic birds, but many animals are susceptible to infection, including swine and humans. While humans are not readily infected with avian influenza viruses, in rare cases direct avian to human transmission has occurred. Swine are readily susceptible to avian, swine, and human influenza subtypes and provide a vessel for genome reassortment among different subtypes of the virus. The influenza A virus genome is made up of eight negative sense RNA segments (vRNA). Reassortment of genome segments between different influenza A subtypes can yield new influenza A subtypes that have potential to cause a human influenza pandemic. While the 1918 pandemic virus was found to be of wholly avian origin, the

\footnotetext{
* Correspondence: Inewcomb@.csusb.edu

Department of Biology, California State University San Bernardino, 5500 University Parkway, San Bernardino, CA 92407, USA
}

1957 and 1968 pandemics contained segments of avian and human origin [1]. In this case it is unclear if swine or human were the vessel of reassortment; although swine are more readily infected with avian influenzas, thus providing more opportunity for reassortment, humans can be infected with avian influenza, and although rare, reassortment within a human host remains a possibility [1]. The 2009 influenza H1N1 pandemic contained segments of avian, swine, and human origin and was thus a triple reassortant that likely emerged from swine [2].

Influenza infection is typically prevented by annual vaccination. However, vaccines are not useful after infection or against emerging subtypes of influenza not targeted during vaccine production, as witnessed with the novel H1N1 pandemic in 2009. Therefore, antivirals that target specific proteins to inhibit virus replication are necessary to stave the spread of an emerging pandemic. The eight genome segments of influenza A virus encode 10 different coding mRNAs by way of alternate splicing [3] which result in over 12 proteins due to alternate translation [4-7]. Two viral segments code for the surface proteins HA and NA for which influenza subtypes are named. Three viral gene segments encode the RNA 
dependent RNA polymerase complex: PA, PB1, and PB2. At least two of these genome segments also encode alternate translation products including PB1-F2, PB1-N40, PA-X, PA-N155 and PA-N182 [4-7]. One segment encodes the nucleoprotein NP. Two segments, $\mathrm{M}$ and NS, undergo alternate splicing to produce NS1, NS2 (NEP), M1 and M2 proteins. With so many viral protein interactions required in various stages of the influenza life cycle, there are numerous potential target sites for antiviral treatments. Current antivirals target the activities of M2 and NA, but resistance is emerging. This review catalogs the current state of influenza antiviral resistance and describes promising new molecules targeting proteins within the viral ribonucleoprotein (vRNP), the complex comprised of the viral RNA genome, the RNA-dependent RNA polymerase (RdRP), and nucleoprotein (NP).

\section{Review}

\section{Current antivirals and resistance}

The current antivirals approved by the FDA are, in order of their release, Symmetra (amantadine), Flumadine (rimantadine), Relenza (zanamivir), and Tamiflu (oseltamivir). Amantadine and rimantadine are adamantane derivatives that target and inhibit the M2 ion channel. The M2 ion channel is an integral membrane protein responsible for release of vRNPs during infection [8]. By binding to the M2 ion channel, amantadine and rimantadine inhibit vRNP release and thus viral replication $[9,10]$. The antivirals zanamivir and oseltamivir are NA or neuraminidase inhibitors. NA activity is required to release new virions from infected cells [11]. These drugs inhibit virus release from infected host cells by binding to the active site of the NA protein $[12,13]$.

Resistance against both classes of influenza antiviral treatments has been documented. Resistance to M2 ion channel inhibitors occurs via a triple amino acid deletion at residues $28-31$ or single amino acid substitutions in the transmembrane region spanning residues 26-31 of the M2 protein [14,15]. 100\% of H3N2 influenza A viruses circulating in 2009-2010 and $99.8 \%$ of 2009 pandemic H1N1 were resistant to adamantanes [16]. Many resistant H1N1 isolates encoded V27A substitution, while resistant $\mathrm{H} 3 \mathrm{~N} 2$ isolates were found to encode substitutions at L26F, V27A, A30T, S31N, or G34E [17]. Resistance to adamantanes in H7N9 has also been documented and is acquired by substitution S31N [18].

Resistance to NA inhibitors is less common as this class of inhibitors was developed later. For example, $98.9 \%$ of tested $2009 \mathrm{H} 1 \mathrm{~N} 1$ viruses remained susceptible to oseltamivir, $100 \%$ of 2009 H1N1 viruses tested remained susceptible to zanamivir [19], 100\% of influenza A H3N2 tested remained susceptible to both oseltamivir and zanamivir for the 2012-2013 season [20]. However, multiple single amino acid changes in NA alter susceptibility to the approved neuraminidase inhibitors. Residues V116, I117, E119, Q136, K150, D151, D199, I223, H275, and N295 were selected to monitor for changes that confer drug resistance or reduce efficacy of the antivirals [21]. Resistance evolves during treatment, via single amino acid substitutions including changes to amino acids mentioned above such as E119V, I223R [22,23], and H275Y [24], but also R292K and N294S [25]. Pandemic H1N1 2009 isolates with a substitution at I223R were resistant to neuraminidase inhibitors in addition to M2 ion channel inhibitors as discussed above [23]. Thus, while NA inhibitors are currently still viable to combat most emerging influenza threats, it is only a matter of time before resistance takes hold as with the adamantanes, rendering both current antiviral therapies ineffective against an emerging influenza threat.

Most worrisome is resistance reported among Highly Pathogenic Avian Influenza (HPAI) subtypes that could spur the next pandemic. Avian subtypes such as H5N1, H7N9, and H7N7 have all resulted in human infection. The H5N1 infections result in high morbidity at $~ 60 \%$, while H7N9 and H7N7 have seen more variability in outcome of human infection [26]. Fortunately none of these subtypes have gained the ability to transmit readily from human to human, but unfortunately, these strains already have antiviral resistant isolates reported. For example, all H7N9 isolates tested were resistant to adamantanes via the $\mathrm{S} 31 \mathrm{~N}$ substitution in the $\mathrm{M} 2$ protein [27], while some H7N9 exhibit high resistance to oseltamivir, mid-resistance to peramivir, and low-resistance to zanamivir via the NA R292K substitution [28]. Also of grave concern are $\mathrm{H} 5 \mathrm{~N} 1$ isolates that encode M2 changes to confer resistance to adamantanes and NA changes that reduce susceptibility to neuraminidase inhibitors [29]. With many circulating antiviral resistant strains and the consequences of a looming virulent influenza pandemic, novel antiviral targets must be investigated so that new therapies can be developed before such a catastrophic event occurs. One promising novel viral target is the viral ribonucleoprotein or vRNP. Figure 1 depicts vRNP interactions and activities targeted by new antiinfluenza candidates.

\section{vRNP: viral ribonucleoprotein}

During infection eight vRNPs, containing the eight different vRNA genome segments, are released and imported into the nucleus to transcribe and replicate the vRNA. Transcription of the viral genome proceeds via a cellular capped-mRNA primer cleaved from host mRNA by the viral polymerase [30]. Unlike other RNA genome viruses, which typically replicate in the cytoplasm, influenza must enter the nucleus to steal nascent host capped mRNAs for use as primers in transcription. The polymerase subunit PA provides the endonuclease activity [31,32], while PB2 


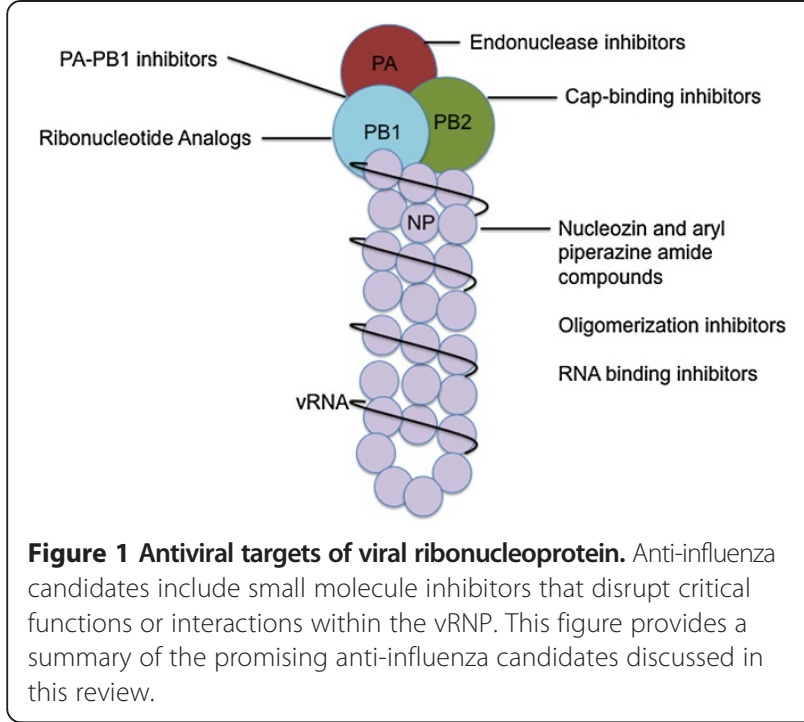

houses the active site where the host pre-mRNA will bind [33]. Polymerization of the viral mRNA transcript proceeds via the $\mathrm{PB} 1$ subunit until the polyadenylation signal of repetitive $\mathrm{U}$ residues result in stuttering by the viral $\mathrm{RdRP}$ and yields the poly(A) tail, terminating transcription [34]. Transcripts of two influenza genome segments, $M$ and NS, undergo alternative splicing to produce M1, M2, NS1 and NS2 (NEP); utilization of host nuclear splicing machinery is another reason for nuclear localization of influenza vRNPs.

Viral replication occurs de novo, without a primer [35]. In the host cell viral RNA replication occurs after translation of viral RdRP and NP proteins. Evidence suggests a model wherein the resident RdRP of the vRNP is responsible for transcription, while a soluble RdRP is responsible for replication from the vRNA template [36]. There are two primer-independent steps of viral replication. First the vRNA template is used to synthesize a full-length complementary (cRNA), which is then replicated to yield progeny vRNA that can be transcribed to mRNA or packaged into new virions during later stages of the viral replication cycle. NP is required for antitermination at the poly $\mathrm{U}$ stretch to allow for replication of full length cRNA [37]. NP encapsidates both cRNA and vRNA replication products and is necessary for genome length functional cRNPs and vRNPs, respectively [38].

\section{NP: nucleoprotein}

The crystal structure of NP [39] (Figure 2) reveals two regions termed the body domain and the head domain, between which lies a deep groove comprised of positively charged basic amino acid residues that form ionic bonds with the negatively charged phosphate backbone of viral cRNA and vRNA. On the opposing side of the RNA binding groove lies a tail loop for oligomerization

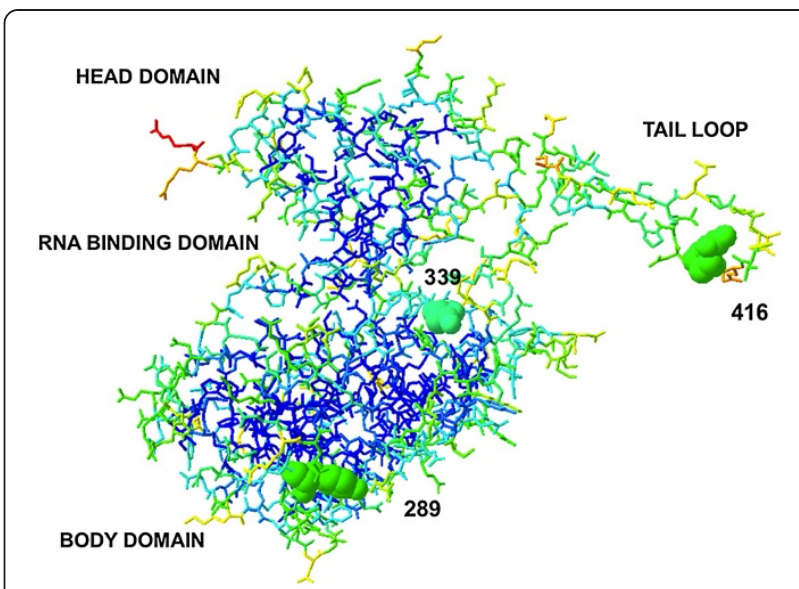

Figure 2 Antiviral targets of nucleoprotein. Analysis of NP monomer crystal structure extracted from NP trimer crystal structure "2IQH" [39] using Deep View-Swiss-PdbViewer 4.0. Residue color determined by accessibility. Greatest to least accessible as follows: red, orange, yellow, green, light blue, and dark blue. Residue 289 is the proposed site of interaction with the antiviral compound nucleozin [41]. An intermolecular salt bridge formed by residues 339 and 416 of NP is essential for oligomerization and can be disrupted by small molecule inhibitors [42].

with other NP monomers. The tail loop of NP spans residues 402-428, and is critical to NP oligomerization and RNA binding, as shown through a tail loop deletion mutant that produced primarily monomeric NP unable to oligomerize and bind RNA [40]. Another crucial oligomerization interaction occurs through a salt bridge between residue 339 of one NP monomer and residue 416 of another NP monomer $[39,41,42]$. Mutational studies disrupting this salt bridge led to inhibition of viral RNA synthesis in vitro, further highlighting the importance of the interaction between NP molecules for vRNP function [42].

However, NP is more than a structural RNA binding protein. NP associates with viral proteins such as PB1 and PB2 [43], and M1 [44], in addition to several cellular factors [45]. Interaction between NP and the RdRP enhanced unprimed replication in vitro, suggesting the NP-RdRP interaction may regulate the switch from primer initiated transcription to unprimed replication [46]. Interaction of NP with the polymerase subunits was crudely mapped to regions within both the body and head domain of the NP crystal structure [43,39]. NP-PB2 and NP-PB1 associations were confirmed through coimmunoprecipitation assays in the absence of other viral factors [43]. The NP-PB2 interaction was refined to residue 627 and 630 of PB2 and residue 150 of NP, with the strength of NP-PB2 interaction directly correlated with RdRP activity [47]. Residue R150 of NP is highly conserved and vital for normal viral RNA synthesis during influenza A WSN infection of MDCK cells [48]. As discussed below, PB2 residue 627 is a well-characterized host range determinant [49,50]. Additional NP mutational 
analysis revealed alanine substitutions within three residues of the head domain at 204, 207, and 208 disrupt interaction with the viral polymerase and inhibit viral RNA synthesis [51]. Cryo-EM and cryo-electron tomography data of native vRNPs in packaged virions revealed two different conformations of polymerase interaction with NP within the vRNP [52]. Together, the evidence points to multiple residues on NP that participate in NP$\mathrm{RdRP}$ interactions. The protein sequence homology of NP is a staggering ninety percent among influenza $\mathrm{A}$ isolates [53], with the described interaction domains exhibiting even greater homology, highlighting the potential of disrupting NP interactions as an effective antiviral target.

\section{RdRP: RNA dependent RNA polymerase}

The influenza A virus RNA dependent RNA polymerase is a heterotrimer comprised of PA, PB2, and PB1.

\section{$P A$}

While PA is required for both viral transcription and replication, the major role attributed to PA during influenza infection is the endonuclease activity needed to steal capped primers for viral transcription initiation in the nucleus of a host cell [30-32,54]. Several highly conserved endonuclease active sites span the N-terminal 209 residues of PA [32]. Alanine screening of amino acids 102 through 134 revealed residues necessary for endonuclease activity [55]. Substitutions D108A and K134A individually inhibited both endonuclease activity and transcription in vitro [31,55]. PA also makes contacts with both vRNA and cRNA promoters between residues 100-180, though the precise residues involved are debated [55-57]. In addition, PA has documented protease activity with Ser 624 defined as the active site [55], though the purpose of this activity remains poorly understood. The essential endonuclease activity of PA is an excellent target for antivirals.

\section{PB2}

PB2 contains the cap-binding domain, which recognizes the capped structure on nascent host mRNAs to be cleaved by the endonuclease site of PA [33]. PB2 residues 318-483 comprise this domain and contain two aromatic amino acids at positions 363 and 404 necessary for cap-binding $[58,59]$. The cap-binding domain of PB2 may also mediate interaction between PB1 and PB2, specifically a loop consisting of residues 421-427 of PB2, which is essential for cap-dependent transcription but not cap-binding, as determined by a deletion mutant [59]. Cap-binding and capped RNA primed transcription are essential activities for influenza that can be targeted by novel antivirals.

PB2 also makes contacts with both vRNA and cRNA promoters in an alpha helix rich region between residues
535-684, which form an RNA binding domain [50]. Located within this domain is residue 627, a wellcharacterized species and pathogenicity determinant for influenza A viruses [49,50,60-63]. Avian influenza A viruses encode PB2 with glutamic acid at residue 627, while human influenza A viruses encode lysine [49]. RNA binding ability of PB2 was linked to the amino acid encoded at 627, with increased RNA binding shown for PB2 proteins containing K627 [50]. Polymerase activity and interaction of PB2 with NP were also shown to be influenced by PB2 residue 627, in addition to residue 630 [47]. Characteristic avian PB2 residue E627 must also encode R630 for proper polymerase activity and coimmunoprecipitation with NP [47]. Characteristic human PB2 residue K627 requires G630 for proper polymerase activity and co-immunoprecipitation with NP [47]. Residue 627 of PB2 influences binding of NP and RNA, two interactions essential for viral RNA synthesis, making this region of $\mathrm{PB} 2$ a favorable target for new antivirals.

\section{PB1}

PB1 is the RNA polymerizing subunit of the RNA dependent RNA polymerase. Residues 1-83 and 494757 of PB1 contribute to vRNA template interaction through in vitro analysis of PB1 deletion mutants [64]. PB1 interacts with PA through its $\mathrm{N}$ terminal domain and PB2 through its $\mathrm{C}$ terminal domain, thus forming the functional RNA dependent RNA polymerase [65-68]. RNA dependent RNA polymerase activity of RNA viruses represents a viral activity that can be targeted by antivirals.

The C-terminal domain of PB1 (678-757) and the Nterminal domain of PB2 (1-37) were defined as the regions responsible for PB1-PB2 interaction and were crystalized to facilitate further investigation $[66,69]$. Crystal structure of this interaction reveals all contacts occur through helix 1 of PB2 (residues 1-12), which is essential for proper RNA polymerase activity [69]. The PB1-PB2 protein interface is of great interest as an antiviral target due to the conservation of these domains in both human and avian viruses [69].

The N-terminus of PB1 (1-80) interacts with the C terminal region of PA consisting of residues 239-716 $[66,70]$. Interaction surfaces of both PB1 and PA are highly conserved $[68,71,72]$. The crystal structure of this interaction reveals PB1 N terminal 25 residues occupy a C-terminal hydrophobic groove of PA [68] (Figure 3). The C-terminal domain of PA has been referred to as a "dragon's head" that holds the N-terminus of PB1 in its "jaws" [68]. A peptide analog of the N-terminal 25 amino acids of PB1 blocks formation of the RNA dependent RNA polymerase complex resulting in no viral replication [68,73]. These studies demonstrate the 


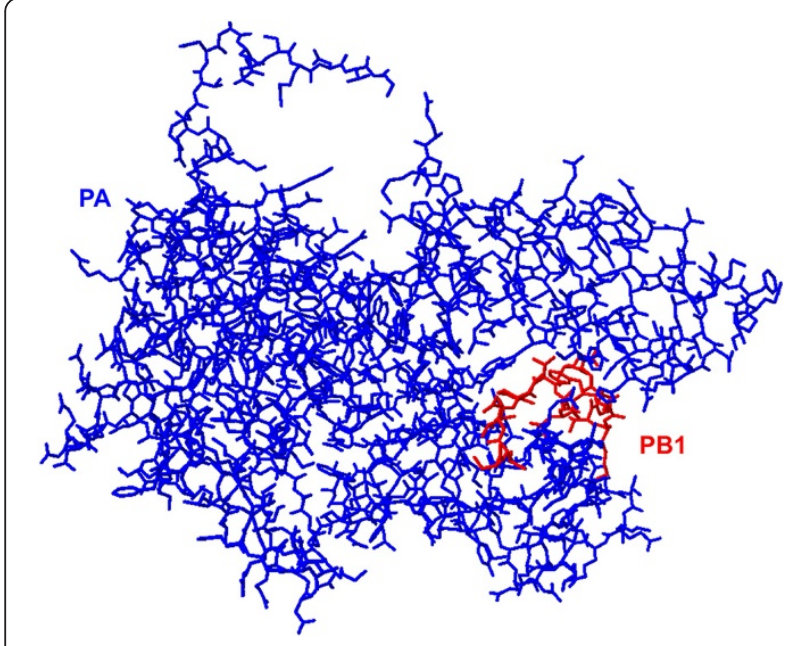

Figure 3 PA-PB1 interaction site is an antiviral target. Analysis of the $P A_{C}-P B 1_{N}$ Crystal structure "3CM8" [68] using Deep View-SwissPdbViewer 4.0. Residues 1-16 of PB1 (red) are shown in interaction with residues 258-716 of PA (blue). Many small molecule inhibitors of PA resemble the $\mathrm{N}$ terminus of $\mathrm{PB} 1$ and bind in the hydrophobic pocket blocking essential interactions between the polymerase subunits $[68,73,95]$.

critical interaction between PB1 and PA in the formation of the RNA dependent RNA polymerase heterotrimer vital for viral RNA synthesis, making this interaction a potential target for novel antivirals.

\section{New antivirals targeting influenza vRNP}

The critical roles of the influenza vRNP for viral RNA synthesis make activities of the vRNP, such as capsnatching and RNA polymerization, excellent antiviral targets. A recently discovered nucleotide analog preferentially utilized by viral RNA dependent RNA polymerases including influenza vRNP, is under study as a promising antiviral therapy targeting the activity of viral RNA dependent RNA polymerases [74]. Further, the multiple essential interactions of the vRNP, such as with each other to form the RNA dependent RNA polymerase heterodimer, with host capped mRNAs to obtain primers for viral transcription, and with NP to regulate and enhance RNA replication, coupled with high conservation of these domains among influenza subtypes, make the proteins of the vRNP excellent targets for small molecule inhibitors with broad efficacy against multiple influenza A subtypes.

\section{Ribonucleotide analogs}

Favipiravir is a 6-fluoro-3-hydroxy-2-pyrazinecarboxamide molecule (also known as T-705) that upon phosphorylation becomes favipiravir-ribofuranosyl-5'-triphosphate (RTP) and inhibits many viral RNA dependent RNA polymerases [75]. Favipiravir is effective against influenza A, influenza B, influenza $\mathrm{C}$, hantaviruses, flaviviruses, noroviruses, and most recently ebola viruses [74-76]. The T-705 RTP is erroneously interpreted as a purine nucleotide by the viral polymerase during RNA elongation [75,77]. Once incorporated into the elongating viral RNA, the analog may hinder strand extension [77]. The antiviral activity of Favipiravir includes influenza $\mathrm{A}(\mathrm{H} 3 \mathrm{~N} 2), \mathrm{A}(\mathrm{H} 1 \mathrm{~N} 1), \mathrm{A}(\mathrm{H} 5 \mathrm{~N} 1), \mathrm{A}(\mathrm{H} 7 \mathrm{~N} 9)$, and strains bearing resistance to both classes of the current FDA approved influenza antivirals [74,75,78].

The $50 \%$ inhibitory concentration $\left(\mathrm{IC}_{50}\right)$ of favipiravir for influenza, determined by plaque reduction assay, was 0.013-0.48 $\mu \mathrm{g} / \mathrm{ml}$ with no cytotoxic effect up to $1000 \mu \mathrm{g} /$ $\mathrm{ml}$ [74]. Human DNA polymerase $\alpha, \beta$, or $\gamma$ with $1000 \mu \mathrm{M}$ of favipiravir showed little sign of inhibition [79] and human RNA polymerase II had an $\mathrm{IC}_{50}$ of $905 \mu \mathrm{M}$ of favipiravir [80]. Therefore, the $\mathrm{IC}_{50}$ of host polymerases is well over 2000 times greater then the $\mathrm{IC}_{50}$ for influenza vRNP, making favipiravir highly selective for influenza vRNP [75]. Favipiravir for influenza therapy has finished two Phase II clinical trials in the United States and one Phase III clinical trial in Japan [75]. Favipiravir is a favorable candidate for a broadly effective antiviral therapy targeting RNA viruses with RNA dependent RNA polymerases that preferentially incorporate favipiravir in RNA synthesis. It is not yet clear if or how quickly influenza vRNP will evolve resistance to this nucleotide analog, but based on HBV and approved nucleotide analog therapies [81], as with any antiviral therapy, if resistance is possible, it will eventually develop, stressing the need to consistently look for novel antiviral targets and therapies.

\section{Small molecule inhibitors}

Unlike nucleotide analog therapies, small molecule inhibitors work by interaction with a viral protein to block a function or interaction and inhibit viral replication. Crystal structure data provides much information to identify small molecules with potential to bind a domain of the target protein and inhibit essential functions or interactions. Small molecules that target conserved regions are likely to have the best efficacy against multiple subtypes. Further, conserved regions are less likely to tolerate mutation and evolve a viable virus with resistance to the small molecule.

\section{Targeting NP}

Nucleozin is a small molecule inhibitor of NP that works by promoting NP oligomerization, blocking nuclear entry through aggregation of NP molecules at the nuclear membrane [41] and inducing vRNP aggregation during cytoplasmic trafficking [82]. Molecular docking models identified two proposed nucleozin interaction sites at residue 289 and 309 of NP [41]. Both residues participate to stabilize the interaction; a tyrosine at residue 289 forms aromatic ring stacking with nucleozin, while an asparagine at residue 309 shares a hydrogen 
bond with nucleozin. Strains found to be nucleozin resistant encoded a histidine in place of a tyrosine at residue 289 of NP [41]. From the crystal structure of NP, residue 289 is relatively accessible to interact with nucleozin (Figure 2) $[39,41]$. The $289 \mathrm{H}$ NP variant likely disrupts a critical point of interaction between NP and nucleozin. Sequence analysis of the NP gene of 3,881 influenza strains revealed $\mathrm{Y} 289 \mathrm{H}$ substitution in 527 strains. Unfortunately the $\mathrm{A}(\mathrm{H} 1 \mathrm{~N} 1) \mathrm{pdm} 09$ strain was shown to contain this single amino acid alteration, signifying it as a nucleozin resistant strain [41]. Nucleozin demonstrated a $50 \%$ effective concentration $\left(E_{50}\right)$ of only $0.069 \mu \mathrm{M}$ against influenza with $50 \%$ cytotoxic concentration greater than $250 \mu \mathrm{M}$ [41]. Thus nucleozin still holds potential as a potent antiviral for strains of influenza housing a tyrosine at residue 289 [41]. In addition to nucleozin, there are several other aryl piperazine amide compounds discovered in parallel that target NP and inhibit virus replication in the same manner [41,83-85]. Cianci et al. provide a detailed review into the efficacy of aryl piperazine amides, including nucleozin, as NP inhibitors [85]. Further analysis of these compounds could lead to the synthesis of an optimized aryl piperazine amide inhibitor of viral replication, even against the circulating nucleozin resistant strains of influenza.

The essential salt bridge for NP oligomerization at residues 339 and 416 was targeted for disruption by small molecule inhibitors (Figure 2) [42]. Peptides that mimicked the NP tail loop (residues 402-428) bound in the tail loop binding pocket and inhibited NP oligomerization, resulting in decreased viral replication by greater than fifty percent [42]. Random virtual screening of small molecules identified four compounds (termed \# 3, 7, 12, and 23) that interrupt NP oligomerization and decrease viral replication [42]. The $\mathrm{IC}_{50}$ of compounds $3,7,12$, and 23 ranged from 2.4 to $118 \mu \mathrm{M}$ in cells challenged with influenza A/WSN/33 for nine hours at a multiplicity of infection (MOI) of 0.2 [42]. Although the targeted NP domain is highly conserved, more research will need to be carried out to ensure little to no development of resistance to the compounds. Importantly, cytotoxicity of these compounds will need to be assessed before they can be of use as antiviral therapies.

Mycalamide A is an antiviral and antitumor compound isolated from Mycale sponges but is unfortunately toxic to cells [86]. A photo-cross-linked chemical array identified analogs of mycalamide A that possess the ability to bind NP [87]. One compound inhibited viral replication up to $77 \%$ in a plaque assay using influenza virus (A/ WSN/33) with no cytotoxic effect [87]. Binding affinity of the analogs was greatest within the N-terminal 110 amino acids of NP [87]. Although the mechanism of inhibition remains uncharacterized, the $\mathrm{N}$-terminus of NP contains an important non-canonical nuclear localization signal (NLS) $[88,89]$ and interacts with host RNA processing factors UAP56 and URH49 $[90,91]$ proposed to enhance RNA replication [92]. More research is needed on these compounds to elucidate the mechanism of inhibition and determine if or how quickly influenza NP will evolve resistance.

Naproxen is an over the counter nonsteroidal antiinflammatory drug that inhibits NP from associating with RNA in the RNA binding groove (Figure 2) [93]. From several molecular docking studies, residues Y148, Q149, R150, R355, R361, and F489 are believed to stabilize naproxen binding to NP [93]. Naproxen acts selectively upon monomeric NP and was shown to protect MDCK cells and mice from a viral challenge at an MOI of $10^{-2}-10^{-3}$ or $50-2,000$ PFU respectively, with little to no cytotoxic effects [93]. Subtypes of H1N1 and H3N2 were both susceptible to inhibition and treatment resulted in efficient protection from viral challenge with either subtype [93]. No escape mutant viruses were produced in response to $500 \mu \mathrm{M}$ naproxen treatment in cells after six passages [93]. Within this experiment the mode of delivery for naproxen was intraperitoneal injection or intranasal treatment in mice that displayed an $\mathrm{EC}_{50}$ of $40 \mathrm{mg} / \mathrm{kg}$ [93]. Naproxen is currently used as an oral medication with a recommended dosage of $220 \mathrm{mg}$ every 8 hours for pain. Naproxen is not currently used as an antiviral but could be optimized for antiviral use through further experimentation and improved drug design.

\section{Targeting PA-PB1 interaction}

Inhibitors of the PA-PB1 interaction are numerous [94]. Compound 1 was discovered through in silico screening [94] using the crystal structure of $\mathrm{PA}_{C}-\mathrm{PB}_{\mathrm{N}}$ (Figure 3) [68]. The hydrophobic pocket of PA houses compound 1 according to molecular docking studies, and inhibition of PA-PB1 interaction was demonstrated through ELISA and immunoprecipitation of PA [94]. Compound 1 inhibited RNA polymerase activity in a dose-dependent manner with an $\mathrm{IC}_{50}$ of about $18 \mu \mathrm{M}$ assessed in a minireplicon assay using a firefly luciferase reporter gene, with no significant cytotoxic effect up to concentrations of 250-1000 $\mu \mathrm{M}$ [94]. Compound 1 inhibited viral replication in MDCK cells for several influenza A H1N1 and H3N2 strains, a swine-origin influenza virus, and an oseltamivir-resistant isolate, with $\mathrm{IC}_{50}$ ranging from 12.2 to $22.5 \mu \mathrm{M}$ [94].

There are two FDA approved medications that in addition to their intended use also possess anti-IAV abilities due to their structural similarity with the $\mathrm{N}$ terminal domain of PB1 that interacts with the C-terminus of PA (Figure 3) [95]. Benzbromarone is approved to 
treat gout and hyperuricemia by promoting the excretion of uric acid. Diclazuril is most commonly used in veterinary medicine as an anti-coccidial. Although these drugs are FDA approved, appropriate drug dosages for use against viral challenges would need to be established before they could be employed for use against influenza A. Testing for the ability of viruses to gain resistance to these drugs must also be done. Benzbromarone and diclazuril could potentially be utilized if an influenza strain arises that possesses resistance to other antivirals available [95].

In addition, a compound derived from licorice, 18 $\beta$ glycyrrhetinic acid (GHA), is a naturally occurring compound that exhibits some anti-IAV activity attributed to interaction with the C terminal domain of PA [96]. Molecular docking studies identified GHA as a ligand to $\mathrm{PA}_{\mathrm{C}}$ [96]. GHA decreased polymerase activity $80 \%$, as assessed by a primer extension assay for cRNA synthesis [96]. These preliminary findings need to be investigated further with more informative assays including in vitro PA-PB1 interaction inhibition study, in vivo polymerase activity assays in tissue culture, and in vivo infection in an animal model. There are many small molecules that mimic the $\mathrm{N}$ terminus of $\mathrm{PB} 1$ fit in the hydrophobic groove of PA to inhibit PA-PB1 interaction (Figure 3) and should be studied further for their potential as an anti-influenza A treatment.

\section{Targeting PA endonuclease}

Fullerene $\left(C_{60}\right)$ is a spherical molecule of sixty carbon atoms that exhibits anti-influenza activity [97]. Full length PA and an isolated PA endonuclease domain were tested in an in vitro endonuclease assay in the presence of fullerene derivatives. Seven fullerene derivatives were able to inhibit the endonuclease ability of the full length PA and isolated endonuclease domain [97]. Docking simulations reveal the fullerene skeleton fits nicely into the endonuclease domain active pocket [97]. MDCK cells were infected with influenza A H1N1 or H3N2 mixed with 0 to $100 \mu \mathrm{M}$ fullerene derivative and immunostained for NP at 24 hours post infection to reveal significantly less NP in cells infected with virus pre-incubated with fullerene compared to a DMSO control [97]. Twelve derivatives of fullerene were tested and resulted in varying efficacy against influenza but showed no cytotoxic effect up to $100 \mu \mathrm{M}$ [97]. More investigation into the activity and expression of viral proteins in response to fullerene needs to be conducted. Importantly, treatment applied post-infection needs to be investigated. This novel compound exemplifies yet another possible antiviral target within the vRNP.

\section{Targeting PB2 cap-binding domain}

Compounds that mimic the 7-methylguanosine moiety of the 5' cap of mRNAs may serve as transcriptional inhibitors. Docking studies of the cap-binding domain of PB2 revealed 7-alkylguanine derivatives as potential inhibitory compounds [98]. Several compounds bind the cap-binding domain of PB2 with greater affinity than a biotinylated cap analogue [98]. These compounds should be further studied and optimized for anti-influenza activity.

\section{Conclusion}

Influenza A virus continues to remain a human menace, in terms of both human health and global economic costs. The wide host range of influenza A virus, coupled with a lack of proofreading activity within the viral RNA dependent RNA polymerases, and a segmented RNA genome allowing for segment reassortment, provide influenza A virus with the ability to evolve rapidly. While yearly vaccination is protective against the strains and subtypes predicted to be circulating and represented during vaccine production, vaccination will not protect against an unseen, emerging subtype of the virus. Antivirals are the first line of defense for an emerging pandemic and resistance to current antivirals is already circulating within influenza A viruses, hastening the resolve to identify new antiviral therapies. The viral ribonucleoprotein (vRNP) is essential for viral replication, making it an ideal target for antivirals. Essential activities of the vRNP include cap-snatching activity required for viral mRNA transcription and RNA polymerase activity required for viral mRNA transcription and RNA replication. The interactions required to form functional vRNPs with these essential activities comprise the most highly conserved protein domains within influenza A subtypes. These interaction domains represent ideal targets for small molecule inhibitors, as these domains are less likely to tolerate mutations. Combination drug therapy is also a potential means to challenge emerging antiviral resistance. The vRNP provides multiple viral protein targets to reduce selection pressures and emergence of resistant strains. However, if mutations conferring antiviral resistance are tolerated, history dictates these mutations will be selected by use of the antiviral and propagate in circulating influenza A viruses. This means the search for new influenza A antiviral inhibitors should be ongoing until a universal vaccine is achieved.

\section{Competing interests}

The authors declare that they have no competing interests.

\section{Authors' contributions}

AMD initiated the review and wrote the initial draft of the paper, which was built upon and edited. AMD prepared the figures. AMD researched and prepared VRNP and new antiviral sections. BJC researched and prepared the state of current antiviral resistance section. AMD and LLN contributed equally to the background. LLN provided the abstract, conclusion, and extensive editing of the final draft. All authors read and approved the final manuscript. 


\section{Authors' information}

AMD is a master's graduate student at CSUSB conducting MS thesis research focused on the role of influenza A virus NP for viral RNA synthesis and gene expression in the laboratory of LLN. BJC is an undergraduate at CSUSB. LLN is Associate Professor of Biology at CSUSB and PI for influenza A research projects in the laboratory.

\section{Acknowledgements}

The authors would like to thank Dr. Stuart Sumida for inspiring AMD to write this review article. The authors would also like to acknowledge the National Institutes of Health grant SC3GM099559 awarded to LLN for supporting research related to this review.

\section{Received: 26 June 2014 Accepted: 12 September 2014}

Published: 16 September 2014

\section{References}

1. Reid $A H$, Taubenberger JK: The origin of the 1918 pandemic influenza virus: a continuing enigma. J Gen Virol 2003, 84:2285-2292.

2. Neumann G, Noda T, Kawaoka Y: Emergence and pandemic potential of swine-origin H1N1 influenza virus. Nature 2009, 459:931-939.

3. Dubois J, Terrier O, Rosa-Calatrava M: Influenza viruses and mRNA splicing: doing more with less. mBio 2014, 5:e00070-14.

4. Chen W, Calvo PA, Malide D, Gibbs J, Schubert U, Bacik I, Basta S, O'Neill R, Schickli J, Palese P, Henklein P, Bennink JR, Yewdell JW: A novel influenza A virus mitochondrial protein that induces cell death. Nat Med 2001, 7:1306-1312.

5. Wise HM, Foeglein A, Sun J, Dalton RM, Patel S, Howard W, Anderson EC, Barclay WS, Digard P: A complicated message: identification of a novel PB1-related protein translated from influenza A virus segment 2 mRNA. J Virol 2009, 83:8021-8031.

6. Jagger BW, Wise H, Kash JC, Walters KA, Wills NM, Xiao YL, Dunfee RL, Schartzman LM, Ozinsky A, Bell GL, Dalton RM, Lo A, Efstathiou S, Atkins JF, Firth $A E$, Taubenberger JK, Digard $P$ : An overlapping protein-coding region in influenza A virus segment 3 modulates the host response. Science 2012, 337:199-204.

7. Muramoto Y, Noda T, Kawakami E, Akkina R, Kawaoka Y: Identification of novel influenza A virus proteins translated from PA mRNA. J Virol 2012, 87:2455-2462

8. Pinto $L H$, Holsinger $L$, Lamb RA: Influenza virus $M_{2}$ protein has ion channel activity. Cell 1992, 69:517-528.

9. Hay AJ, Wolstenholm AJ, Skehel JJ, Smith MH: The molecular basis of the specific anti-influenza action of amantadine. EMBO J 1985, 4:3021-3024

10. Jing X, Ma C, Ohigashi Y, Oliveria FA, Jardetzky TS, Pinto LH, Lamb RA: Functional studies indicate amantadine binds to the pore of the influenza A virus M2 proton-selective ion channel. Proc Natl Acad Sci U S A 2008, 105:10967-10972.

11. Davis AR, Bos TJ, Nayak DP: Active influenza virus neuraminidase is expressed in monkey cells from cDNA cloned in simian virus 40 vectors. Proc Natl Acad Sci U S A 1983, 80:3976-3980.

12. Meindl $P$, Bodo G, Palese $P$, Schulman J, Tuppy $H$ : Inhibition of neuraminidase activity by derivatives of 2-deoxy-2,3-dehydro-N-acetylneuraminic acid. Virology 1974, 58:457-463.

13. Palese $P$, Compans RW: Inhibition of influenza and parainfluenza virus replication in tissue culture by 2-deoxy-2, 3-dehydro-N-trifluoracetylneuraminic acid (FANA). Virology 1974, 59:490-498.

14. Holsinger $L$, Nichani $D$, Pinto LH, Lamb RA: Influenza $A$ virus $M 2$ ion channel protein: a structure-function analysis. J Virol 1984, 68:1551-1563.

15. Abed Y, Goyette N, Boivin G: Generation and characterization of recombinant influenza $A(\mathrm{H} 1 \mathrm{~N} 1)$ viruses harboring amantadine resistance mutations. Antimicrob Agents Chemother 2005, 49:556-559.

16. Centers for Disease Control and Prevention. http://www.cdc.gov/mmwr/ preview/mmwrhtml/mm5929a2.htm.

17. Tang JW, Ngai KL, Wong JC, Lam WY, Chan PK: Emergence of adamantane-resistant influenza A (H3N2) viruses in Hong Kong between 1997 and 2006. J Med Virol 2008, 80:895-901.

18. Chen Y, Liang W, Yang S, Wu N, Gao H, Sheng J, Yao H, Wo J, Fang Q, Cui D, Li Y, Yao X, Zhang Y, Wu H, Zheng S, Diao H, Xia S, Zhang Y, Chan KH, Tsoi HW, Teng JL, Song W, Wang P, Lau SY, Zheng M, Chan JF, To KK, Chen $H$, Li L, Yuen KY: Human infections with the emerging avian influenza $A$
H7N9 virus from wet market poultry: clinical analysis and characterisation of viral genome. Lancet 2013, 381:1916-1925.

19. Centers for Disease Control and Prevention. http://www.cdc.gov/flu/ pastseasons/0910season.htm.

20. Centers for Disease Control and Prevention. http://www.cdc.gov/flu/ about/qa/antiviralresistance.htm.

21. Deyde VM, Sheu TG, Trujillo AA, Okomo-Adhiambo M, Garten R, Klimov Al, Gubareva LV: Detection of molecular markers of drug resistance in 2009 pandemic influenza $A(\mathrm{H} 1 \mathrm{~N} 1)$ viruses by pyrosequencing. Antimicrob Agents Chemother 2010, 54:1102-1110.

22. Pizzorno A, Abed Y, Bouhy X, Beaulieu É, Mallett C, Russell R, Boivin G: Impact of mutations at residue 1223 of the neuraminidase protein on the resistance profile, replication level, and virulence of the 2009 pandemic influenza virus. Antimicrob Agents Chemother 2012, 56:1208-1214.

23. van der Vries E, Kroeze EJV, Stittelaar KJ, Linster M, Van der Linden A Schrauwen EJ, Leijten LM, van Amoerogen G, Schutten M, Kuiken T, Osterhaus AD, Fouchier RA, Boucher CA, Herfst S: Multidrug resistant 2009 A/H1N1 influenza clinical isolate with a neuraminidase I223R mutation retains its virulence and transmissibility in ferrets. PLOS pathogens 2011, 7:e1002276.

24. Baz M, Abed Y, Papenbug J, Bouhy X, Hamelin ME, Boivin G: Emergenge of oseltamivir-resistant pandemic H1N1 virus during prophylaxis. Engl J Med 2009, 361:2296-2297.

25. Kiso M, Mitamura K, Sakai-Tagawa Y, Shiraishi K, Kawakami C, Kimura K, Hayden FG, Sugaya N, Kawaoka Y: Resistant influenza A viruses in children treated with oseltamivir: descriptive study. Lancet 2004, 364:759-765.

26. WHO. http://www.who.int/influenza/human_animal_interface/ avian_influenza/h5n1_research/faqs/en/.

27. Gao R, Cao B, Hu Y, Feng Z, Wang D, Hu W, Chen J, Jie Z, Qiu H, Xu K, Xu X, Lu H, Zhu W, Gao Z, Xiang N, Shen Y, He Z, Gu Y, Zhang Z, Yang Y, Zhao X, Zhou L, Li X, Zou S, Zhang Y, Yang L, Guo J, Dong J, Li Q, Dong L, et al. Human infection with a novel avian-origin influenza $A$ (H7N9) virus. N Engl J Med 2013, 368:1888-1897.

28. Hai R, Schmolke M, Leyva-Grado VH, Thangavel RR, Margine I, Jaffe ER, Krammer F, Solòrzano A, Carcia-Sastre A, Palese P, Bouvier NM: Influenza A (H7N9) virus gains neuraminidase inhibitor resistance without loss of in vivo virulence or transmissibility. Nat Commun 2013, 4:2854.

29. Hurt AC, Selleck P, Komadina N, Shaw R, Brown L, Barr IG: Susceptibility of highly pathogenic $A(H 5 N 1)$ avian influenza viruses to the neuraminidase inhibitors and adamantanes. Antivir Res 2007, 73:228-231.

30. Plotch SJ, Bouloy M, Ulmanen I, Krug RM: A unique cap ( ${ }^{7} \mathrm{GpppXm}$ ) dependent influenza virion endonuclease cleaves capped RNAs to generate the primers that initiate viral RNA transcription. Cell 1981, 23:847-858.

31. Yuan P, Bartlam M, Lou Z, Chen S, Zhou J, He X, Lv Z, Ge R, Li X, Deng T, Fodor $E$, Rao Z, Liu Y: Crystral structure of an avian influenza polymerase $P A_{N}$ reveals an endonuclease active site. Nature 2009, 458:909-913.

32. Dias A, Bouvier D, Crépin T, McCarthy AA, Hart DJ, Baudin F, Cusack S, Ruigrok RW: The cap-snatching endonuclease of influenza virus polymerase resides in the PA subunit. Nature 2009, 458:914-918.

33. Li ML, Rao P, Krug RM: The active sites of the influenza cap-dependent endonuclease are on different polymerase subunits. EMBO J 2001, 20:2078-2086.

34. Poon LL, Pritlove DC, Fodor E, Brownlee GG: Direct evidence that the poly (A) tail of influenza A virus mRNA is synthesized by reiterative copying of a U track in the virion RNA template. J Virol 1999, 73:3473-3476.

35. Shapiro Gl, Krug RM: Influenza Virus RNA Replication In Vitro: Synthesis of Viral Template RNAs and Virion RNAs in the Absence of an Added Primer. J Virol 1988, 62:2285-2290.

36. Jorba N, Coloma R, Ortín J: Genetic trans-complementation establishes a new model for influenza virus RNA transcription and replication. PLOS pathogens 2009, 5:e1000462.

37. Beaton $A R$, Krug RM: Transcription antitermination during influenza viral template RNA synthesis requires the nucleocapsid protein and the absence of a 5'capped end. Proc Natl Acad Sci USA 1986, 83:6282-6286.

38. Honda A, Ueda K, Nagata K, Ishihama A: RNA Polymerase of Influenza Virus: Role of NP in RNA Chain Elongation. J Biochem 1988, 104:1021-1026

39. Ye Q, Krug RM, Tao YJ: The mechanism by which influenza A virus nucleoprotein forms oligomers and binds RNA. Nature 2006, 444:1078-1082 
40. Ye Q, Guu TS, Mata DA, Kuo RL, Smith B, Krug RM, Tao YJ: Biochemical and structural evidence in support of a coherent model for the formation of the double-helical influenza A virus ribonucleoprotein. mBio 2012, 4:e00467-12.

41. Kao RY, Yang D, Lau LS, Tsui WH, Hu L, Dai J, Chan MP, Chan CM, Wang P, Zheng BJ, Sun J, Huang JD, Madar J, Chen G, Chen H, Guan Y, Yuen KY: Identification of influenza A nucleoprotein as an antiviral target. Nat Biotechnol 2010, 28:600-605.

42. Shen YF, Chen Y, Chu SY, Lin Ml, Hsu HT, Wu PY, Wu CJ, Liu HW, Lin FY, Lin G, Hsu PH, Yang AS, Cheng YS, Wu YT, Wong CH, Tsai MD: E339... R416 salt bridge of nucleoprotein as a feasible target for influenza virus inhibitors. Proc Nat Acad Sci USA 2011, 108:16515-16520.

43. Biswas SK, Boutz PL, Nayak DP: Influenza virus nucleoprotein interacts with influenza virus polymerase proteins. J Virol 1988, 72:5493-5501.

44. Avalos RT, Yu Z, Nayak DP: Association of influenza virus NP and M1 proteins with cellular cytoskeletal elements in influenza virus-infected cells. J Virol 1997, 71:2947-2958.

45. Portela A, Digard P: The influenza virus nucleoprotein: a multifunctional RNA-binding protein pivotal to virus replication. J Gen Virol 2002, 83:723-734

46. Newcomb LL, Kuo RL, Ye Q, Jiang Y, Tao YJ, Krug RM: Interaction of the influenza a virus nucleocapsid protein with the viral RNA polymerase potentiates unprimed viral RNA replication. J Virol 2009, 83:29-36.

47. Ng AK, Chan WH, Choi ST, Lam MK, Lau KF, Chan PK, Au SW, Fodor E, Shaw PC: Influenza polymerase activity correlates with the strength of interaction between nucleoprotein and PB2 through the host-specific residue K/E627. PLOS ONE 2012, 7:e36415.

48. Li Z, Watanabe T, Hatta M, Watanabe S, Nanbo A, Ozawa M, Kakugawa S, Shimojima M, Yamada S, Neumann G, Kawaoka Y: Mutational analysis of conserved amino acids in the influenza A virus nucleoprotein. J Virol 2009, 83:4153-4162.

49. Subbarao EK, London W, Murphy BR: A single amino acid in the PB2 gene of the influenza A virus is a determinant of host range. J Virol 1993, 67:1761-1764

50. Kuzuhara T, Kise D, Yoshida H, Horita T, Murazaki Y, Nishimura A, Echigo N, Utsunomiya $H$, Tsuge $H$ : Structural basis of the influenza A virus RNA polymerase PB2 RNA-binding domain containing the pathogenicitydeterminant lysine 627 residue. J Biol Chem 2009, 284:6855-6860.

51. Marklund JK, Ye Q, Dong J, Tao YJ, Krug RM: Sequence in the influenza A virus nucleoprotein required for viral polymerase binding and RNA synthesis. J Virol 2012, 86:7292-7297.

52. Arranz R, Coloma R, Chichón J, Conesa JJ, Carrascosa JL, Valpuesta JM, Ortín J, Martĺn-Benito J: The Structure of Native Influenza Virion Ribonucleoproteins. Science 2012, 338:1634-1637.

53. Gorman OT, Bean WJ, Kawaoka Y, Webster RG: Evolution of the nucleoprotein gene of influenza A virus. J Virol 1990, 64:1487-97.

54. Zhao C, Lou Z, Guo Y, Ma M, Chen Y, Liang S, Zhang L, Chen S, Li X, Liu Y, Bartlam M, Rao Z: Nucleoside monophosphate complex structures of the endonuclease domain from the influenza virus polymerase PA subunit reveal the substrate binding site inside the catalytic center. J Virol 2009, 83:9024-9030.

55. Hara K, Schmidt Fl, Crow M, Brownlee GG: Amino acid residues in the $\mathrm{N}$-terminal region of the PA subunit of influenza $\mathrm{A}$ virus RNA polymerase play a critical role in protein stability, endonuclease activity, cap binding, and virion RNA promoter binding. J Virol 2006, 80:7789-7798.

56. Deng T, Vreede FT, Brownlee GG: Different De Novo Initiation Strategies Are Used by Influenza Virus RNA Polymerase on Its CRNA and Viral RNA Promoters during Viral RNA Replication. J. Virol 2006, 80:2337-2348.

57. Maier H, Kashiwagi T, Hara K, Brownlee GG: Differential role of the influenza $A$ virus polymerase PA subunit for vRNA and cRNA promoter binding. Virology 2008, 370:194-204.

58. Fetcher P, Mingay L, Sharps J, Chambers A, Fodor E, Brownlee GG: Two aromatic residues in the PB2 subunit of influenza $A$ RNA polymerase are crucial for cap binding. J Biol Chem 2003, 278:20381-20388.

59. Guilligay D, Tarendeau F, Resa-Infante P, Coloma R, Crepin T, Sehr P, Lewis J, Ruigrok RW, Ortin J, Hart DJ, Cusack S: The structural basis for cap binding by influenza virus polymerase subunit PB2. Nat Struct Mol Bio 2008, 15:500-506

60. Massin P, van der Werf S, Naffakh N: Residue 627 of PB2 is a determinant of cold sensitivity in RNA replication of avian influenza viruses. J Virol 2001, 75:5398-5404.
61. Shinya K, Hamm S, Hatta M, Ito H, Ito T, Kawaoka Y: PB2 amino acid at position 627 affects replicative efficiency, but not cell tropism, of Hong Kong H5N1 influenza A viruses in mice. Virology 2004, 320:258-266.

62. Labadie K, Dos Santos Afonso E, Rameix-Welti MA, Van Der Werf S, Naffakh $\mathrm{N}$ : Host-range determinants on the PB2 protein of influenza A viruses control the interaction between the viral polymerase and nucleoprotein in human cells. Virology 2007, 362:271-282.

63. Tarendeau F, Crepin T, Guilligay D, Ruigrok RW, Cusack S, Hart DJ: Host determinant residue lysine 627 lies on the surface of a discrete, folded domain of influenza virus polymerase PB2 subunit. PLOS Pathog 2008, 4:e1000136

64. González S, Ortin J: Characterization of influenza virus PB1 protein binding to viral RNA: two separate regions of the protein contribute to the interaction domain. J Virol 1999, 73:631-637.

65. Perez DR, Donis RO: A 48-amino-acid region of influenza a virus PB1 protein is sufficient for complex formation with PA. J Virol 1995, 69:6932-6939.

66. González S, Zürcher T, Ortin J: Identification of two separate domains in the influenza virus PB1 protein involved in the interaction with the PB2 and PA subunits: a model for viral RNA polymerase structure. Nucleic Acids Res 1996, 24:4456-4463.

67. Perez DR, Donis RO: Functional analysis of PA binding by influenza a virus PB1: effects on polymerase activity and viral infectivity. J Virol 2001, 75:8127-8136

68. He X, Zhou J, Bartlam M, Zhang R, Ma J, Lou Z, Li X, Li J, Joachimiak A, Zeng Z, Ge R, Rao Z, Liu Y: Crystal structure of the polymerase $\mathrm{PA}_{\mathrm{C}^{-}-\mathrm{PB} 1_{\mathrm{N}}}$ complex from an avian influenza H5N1 virus. Nature 2008, 454:1123-1126.

69. Sugiyama K, Obayashi E, Kawaguchi A, Suzuki Y, Tame JR, Nagata K, Park SY: Structural insight into the essential PB1-PB2 subunit contact of the influenza virus RNA polymerase. EMBO J 2009, 28:1803-1811.

70. Krug RM, Aramini JM: Emerging antiviral targets for influenza A virus. Trends Pharmalcol Sci 2009, 30:269-277.

71. Wunderlich K, Mayer D, Ranadheera C, Holler AS, Mänz B, Martin A, Chase G, Tegge W, Frank R, Kessler U, Schwemmle M: Identification of a PA-binding peptide with inhibitory activity against influenza $A$ and $B$ virus replication. PLOS ONE 2009, 4:e7517.

72. Obayashi E, Yoshida H, Kawai F, Shibayama N, Kawaguchi A, Nagata K, Tame JR, Park SY: The structural basis for an essential subunit interaction in influenza virus RNA polymerase. Nature 2008, 454:1127-1131.

73. Ghanem A, Mayer D, Chase G, Tegge W, Frank R, Kochs G, García-Sastre A, Schwemmel M: Peptide-mediated interference with influenza A virus polymerase. J Virol 2007, 81:7801-7804.

74. Furuta Y, Takahashi K, Fukuda Y, Kuno M, Kamiyama T, Kozaki K, Shiraki K: In vitro and in vivo activities of anti-influenza virus compound T-705. Antimicrob Agents Ch 2002, 46:977-981.

75. Furuta Y, Gowen BB, Takahashi K, Shiraki K, Smee DF, Barnard DL: Favipiravir (T705), a novel viral RNA polymerase inhibitor. Antivir Res 2013, 100:446-454.

76. Oestereich L, Lüdtke A, Wurr S, Rieger T, Muñoz-Fontela C, Gunther S: Successful treatment of advanced Ebola virus infection with T-705 (favipiravir) in a small animal model. Antivir Res 2014, 105:17-21.

77. Jin Z, Smith LK, Rajwanshi VK, Kim B, Deval J: The ambiguous base-pairing and high substrate efficiency of T-705 (Favipiravir) ribofuranosyl 5'-triphosphate towards influenza A virus polymerase. PlOS ONE 2013, 8:e68347.

78. Sleeman K, Mishin VP, Deyde VM, Furuta Y, Klimov Al Gubareva LV: In vitro antiviral activity of favipiravir (T-705) against drug-resistant influenza and 2009 A (H1N1) viruses. Antimicrob. Agents Ch 2010, 54:2517-2524.

79. Kiso M, Takahashi K, Sakai-Tagawa Y, Shinya K, Sakabe S, Le QM, Ozawa M, Furuta Y, Kawaoka Y: T-705 (favipiravir) activity against lethal H5N1 influenza A viruses. Proc Natl Acad Sci USA 2010, 107:882-887.

80. Takahashi K, Smee DF, Shiraki K, Kawaoka Y, Furuta Y: Pharmacological effects of favipiravir, an anti-influenza viral drug. J Med Pharm Sci 2011, 66:429-441.

81. Menéndez-Arias L, Álvarez M, Pacheco B: Nucleoside/nucleotide analog inhibitors of hepatitis $B$ virus polymerase: mechanism of action and resistance. Curr Opin Virol 2014, 8:1-9.

82. Amorim MJ, Kao RY, Digard P: Nucleozin Targets Cytoplasmic Trafficking of Viral Ribonucleoprotein-Rab11 Complexes in Influenza A Virus Infection. J Virol 2013, 87:4694-4703.

83. Su CY, Cheng TJ, Lin MI, Wang SY, Huang WI, Lin-Chu SY, Chen YH, Wu CY, Lai MM, Cheng WC, Wu YT, Tsai MD, Cheng YS, Wong CH: High-throughput 
identification of compounds targeting influenza RNA-dependent RNA polymerase activity. Proc Natl Acad Sci USA 2010, 107:19151-19156.

84. Gerritz SW, Cianci C, Kim S, Pearce BC, Meminie C, Discotto L, McAuliffe B, Minassian BF, Shi S, Zhu S, Zhai W, Pendri A, Li G, Poss MA, Edavettal S, McDonnell PA, Lewis HA, Maskos K, Mörtl M, Kiefersauer R, Steinbacher S, Baldwin ET, Metzler W, Bryson J, Healy MD, Philip T, Zoeckler M, Scartman R, $\operatorname{Sinz} \mathrm{M}$, Leyva-Grado VH, et al. Inhibition of influenza virus replication via small molecules that induce the formation of higher-order nucleoprotein oligomers. Proc Natl Acad Sci USA 2011, 108:15366-15371.

85. Cianci C, Gerritz SW, Deminie C, Krystal M: Influenza nucleoprotein: promising target for antiviral chemotherapy. Antiviral Chem Chemother 2013, 23:77-91.

86. Perry NB, Blunt JW, Munro MH, Pannell LK: Mycalamide A, an antiviral compound from a New Zealand sponge of the genus Mycale. J Am Chem Soc 1988, 110:4850-4851.

87. Hagiwara K, Kondoh Y, Ueda A, Yamada K, Goto H, Watanabe T, Nakata T, Osada H, Aida Y: Discovery of novel antiviral agents directed against the influenza A virus nucleoprotein using photo-cross-linked chemical arrays. Biochem Bioph Res Co 2010, 394:721-727.

88. Wang P, Palese P, O'Neill RE: The NPI-1/NPI-3 (karyopherin alpha) binding site on the influenza a virus nucleoprotein NP is a nonconventional nuclear localization signal. J Virol 1997, 71:1850-1856.

89. Cros JF, García-Sastre A, Palese P: An unconventional NLS is critical for the nuclear import of the influenza $A$ virus nucleoprotein and ribonucleoprotein. Traffic 2005, 6:205-213.

90. Momose F, Basler CF, O'Neill RE, Iwamatsu A, Palese P, Nagata K: Cellular splicing factor RAF-2p48/NPI-5/BAT1/UAP56 interacts with the influenza virus nucleoprotein and enhances viral RNA synthesis. J Virol 2001, 75:1899-908.

91. Wisskirchen C, Ludersdorfer TH, Müller DA, Moritz E, Pavlovic J: The cellular RNA helicase UAP56 is required for prevention of double-stranded RNA formation during influenza A virus infection. J Virol 2011, 85:8646-8655.

92. Kawaguchi A, Momose F, Nagata K: Replication-coupled and host factor-mediated encapsidation of the influenza virus genome by viral nucleoprotein. J Virol 2011, 85:6197-6204.

93. Lejal N, Tarus B, Bouguyon E, Chenevas S, Bertho N, Delmas B, Ruigrok RW, Di Primo C, Slama-Schwok A: Structure-based discovery of the novel antiviral properties of Naproxen against the nucleoprotein of influenza $A$ virus. Antimicrob Agents Ch 2013, 57:2231-2242.

94. Muratore G, Goracci L, Mercorelli B, Foeglein Á, Digard P, Cruciani G, Palù G, Loregian $A$ : Small molecule inhibitors of influenza $A$ and $B$ viruses that act by disrupting subunit interactions of the viral polymerase. Proc Natl Acad Sci USA 2012, 109:6247-6252.

95. Fukuoka M, Minakuchi M, Kawaguchi A, Nagata K, Kamatari YO, Kuwata K: Structure-based discovery of anti-influenza virus A compounds among medicines. Biochim Biophys Acta 1820, 2012:90-95.

96. Li L, Chang SH, Xiang JF, Li Q, Liang HH, Tang YL, Liu YF: NMR identification of anti-influenza lead compound targeting at $\mathrm{PA}_{C}$ subunit of H5N1 polymerase. Chinese Chem Lett 2012, 23:89-92.

97. Shoji M, Takahashi E, Hatakeyama D, Iwai Y, Morita Y, Shirayama R, Echigo N, Kido H, Nakamura S, Mashino T, Okutani T, Kuzuhara T: Anti-influenza Activity of $C_{60}$ Fullerene Derivatives. PLOS ONE 2013, 8:e66337.

98. Pautus S, Sehr P, Lewis J, Fortuné A, Wolkerstorfer A, Szolar O, Guilligay D, Lunardi T, Décout J, Cusack S: New 7-Methylguanine Derivatives Targeting the Influenza Polymerase PB2 Cap-Binding Domain. J Med Chem 2013, 56:8915-8930.

doi:10.1186/1743-422X-11-167

Cite this article as: Davis et al: Emerging antiviral resistant strains of influenza $A$ and the potential therapeutic targets within the viral ribonucleoprotein (VRNP) complex. Virology Journal 2014 11:167.

\section{Submit your next manuscript to BioMed Central and take full advantage of:}

- Convenient online submission

- Thorough peer review

- No space constraints or color figure charges

- Immediate publication on acceptance

- Inclusion in PubMed, CAS, Scopus and Google Scholar

- Research which is freely available for redistribution

Submit your manuscript at www.biomedcentral.com/submit
() Biomed Central 